\title{
Genomic regulation of invasion by STAT3 in triple negative breast cancer
}

Joy M. McDaniel ${ }^{1,2}$, Katherine E. Varley ${ }^{3}$, Jason Gertz ${ }^{3}$, Daniel S. Savic ${ }^{1}$, Brian S. Roberts $^{1}$, Sarah K. Bailey ${ }^{4}$ Lalita A. Shevde ${ }^{4,6}$, Ryne C. Ramaker ${ }^{1,7}$, Brittany N. Lasseigne $^{1}$, Marie K. Kirby ${ }^{1}$, Kimberly M. Newberry ${ }^{1}$, E. Christopher Partridge ${ }^{1}$, Angela L. Jones ${ }^{1}$, Braden Boone ${ }^{1}$, Shawn E. Levy ${ }^{1}$, Patsy G. Oliver ${ }^{5}$, Katherine C. Sexton ${ }^{6}$, William E. Grizzle ${ }^{6}$, Andres Forero ${ }^{6}$, Donald J. Buchsbaum ${ }^{5}$, Sara J. Cooper $^{1}$, Richard M. Myers ${ }^{1}$

${ }^{1}$ HudsonAlpha Institute for Biotechnology, Huntsville, AL 35806, USA

${ }^{2}$ The University of Alabama in Huntsville, Huntsville, AL 35899, USA

${ }^{3}$ Department of Oncological Sciences, Huntsman Cancer Institute, University of Utah, Salt Lake City, UT, 84112, USA

${ }^{4}$ Department of Pathology, University of Alabama at Birmingham, Birmingham, AL, 35294, USA

${ }^{5}$ Department of Radiation Oncology, University of Alabama at Birmingham, Birmingham, AL, 35294, USA

${ }^{6}$ University of Alabama at Birmingham Comprehensive Cancer Center, Birmingham, AL 35294, USA

${ }^{7}$ Department of Genetics, University of Alabama at Birmingham, Birmingham, AL, 35294, USA

Correspondence to: Richard M. Myers, email: rmyers@hudsonalpha.org

Keywords: TNBC, STAT3, ChIP-seq, RNA-seq, invasion

Received: August 15, $2016 \quad$ Accepted: November 14, 2016

Published: December 24, 2016

\section{ABSTRACT}

Breast cancer is a heterogeneous disease comprised of four molecular subtypes defined by whether the tumor-originating cells are luminal or basal epithelial cells. Breast cancers arising from the luminal mammary duct often express estrogen receptor (ER), progesterone receptor (PR), and human epidermal growth receptor 2 (HER2). Tumors expressing ER and/or PR are treated with anti-hormonal therapies, while tumors overexpressing HER2 are targeted with monoclonal antibodies. Immunohistochemical detection of ER, $P R$, and HER2 receptors/proteins is a critical step in breast cancer diagnosis and guided treatment. Breast tumors that do not express these proteins are known as "triple negative breast cancer" (TNBC) and are typically basal-like. TNBCs are the most aggressive subtype, with the highest mortality rates and no targeted therapy, so there is a pressing need to identify important TNBC tumor regulators. The signal transducer and activator of transcription 3 (STAT3) transcription factor has been previously implicated as a constitutively active oncogene in TNBC. However, its direct regulatory gene targets and tumorigenic properties have not been well characterized. By integrating RNA-seq and ChIP-seq data from 2 TNBC tumors and 5 cell lines, we discovered novel gene signatures directly regulated by STAT3 that were enriched for processes involving inflammation, immunity, and invasion in TNBC. Functional analysis revealed that STAT3 has a key role regulating invasion and metastasis, a characteristic often associated with TNBC. Our findings suggest therapies targeting STAT3 may be important for preventing TNBC metastasis.

\section{INTRODUCTION}

Breast cancer is the most prevalent cancer diagnosed in women worldwide, and is the second leading cause of death by cancer in women $[1,2]$. In 2015 , nearly 231,840 new cases of breast cancer in women were diagnosed in the United States, along with an estimated 40,290 female breast cancer-related deaths [2]. The majority of breast cancers diagnosed are ductal invasive carcinomas. Cancers of this type arise from luminal or basal epithelial cells 
lining the mammary duct. Ductal-derived breast cancer can be classified into four categories based on microarray gene expression profiling analysis: luminal A, luminal $\mathrm{B}$, human epidermal growth factor receptor 2 (HER2) overexpression, and basal-like (Basal A and Basal B) [3]. Expression of estrogen receptor (ER), progesterone receptor (PR), and HER2 receptor is used to determine the primary breast tumor subtype, prognosis, and targeted therapeutic regimen.

The presence of ER is one of the most important discriminators for diagnosing primary breast tumor subtype [4]. ER-positive tumors are associated with better prognosis because of available targeted hormonal therapy, longer relapse-free survival, and improved overall survival compared to ER-negative tumors [4-6]. While HER2-positive tumors are aggressive, treatment options for these cancers have advanced through the use of monoclonal antibodies to block HER2 activity [7]. Ten to twenty percent of all invasive breast cancers diagnosed are classified as triple negative breast cancer (TNBC), a subtype characterized by a lack of expression of ER or PR, and a lack of HER2 overexpression [8].

TNBCs typically arise from basal cells, and are diagnosed at higher tumor stage and grade, contributing to the aggressive biology of these cancers [8]. TNBC treatment is restricted to the use of cytotoxic chemotherapies because these tumors are non-responsive to anti-hormonal therapeutics [8]. TNBC patients typically receive neoadjuvant chemotherapy, and in comparison to patients with luminal breast cancers, they have a higher initial response to chemotherapy $[9,10]$. However, when TNBC and non-TNBC patients are compared longitudinally, TNBC patients have shorter relapse-free survival $[9,10]$. TNBC tumors are also more likely to develop resistance to chemotherapies and present with distant recurrence and visceral metastases, all contributing to shorter relapse-free survivals [9-11]. Recurrence typically occurs within 3 years of initial diagnosis [12]. Consequently, in order to advance the development of targeted therapeutics in TNBC, a better understanding of the underlying molecular mechanisms distinguishing TNBC from other breast cancer subtypes is critical.

Genomic studies have reported gene expression signatures characterizing basal TNBC, but lack insight into upstream transcriptional regulators $[3,13,14]$. The high success rate of targeting the transcription factor ER in luminal breast cancers supports the notion that identifying the transcriptional regulator(s) of basal TNBC will be beneficial in the development of therapies for this aggressive breast cancer. Key transcription factors as potential therapeutic targets in basal TNBC are likely to be overexpressed in TNBCs compared to ER+ tumors, and possess known oncogenic mechanisms in other solid tumors. One transcription factor that meets these criteria as a potential therapeutic target in TNBC is signal transducer and activator of transcription 3 (STAT3).
STAT3 has been widely recognized as an oncogene in various cancers and has been confirmed to be constitutively active in TNBC $[15,16]$. There is no difference in the gene expression levels of STAT3 in ER+, HER2, and TNBC breast cancer subtypes; however, active phosphorylated STAT3 is restricted to basal TNBCs [17, 18]. Furthermore, it has been shown that STAT3 signaling is critical for cell survival in basal TNBC breast tumors [18]. As an oncogene, in other cancer types, STAT3 has been shown to regulate various aspects of cancer onset and progression including transformation, proliferation, invasion, and metastasis $[16,19,20]$. Treatment of TNBC cell lines with pharmacological agents targeting STAT3, revealed this factor to be required for cancer stem cell maintenance and cell survival in TNBC $[15,17,18,21]$. These studies implicate a role for STAT3 in the aggressive biology of TNBC; however the transcriptional program associated with STAT3 and its molecular mechanisms are unknown. Here we describe our efforts to characterize the direct regulatory gene targets of STAT3 and its functional role in the progression of basal TNBC.

\section{RESULTS}

\section{Genome-wide binding patterns of STAT3}

To characterize STAT3 binding patterns associated with TNBC, we performed, in replicate, Chromatin Immunoprecipitation followed by massively parallel sequencing (ChIP-seq) in five basal breast cancer cell lines with a STAT3-specific antibody [22]. We identified genomic regions commonly as well as uniquely bound by STAT3 across the breast cancer cell lines. Hierarchical clustering of STAT3 binding sites demonstrated that STAT3 binding is heterogeneous, possibly reflecting the heterogeneity of TNBC (Figure 1). We next evaluated gene enrichment at TNBC-specific STAT3 binding sites using the Genomic Regions Enrichment of Annotations Tool (GREAT) [23], and found these sites were near genes down-regulated in luminal breast cancer and up-regulated in basal breast cancer cell lines (Supplementary Table 1). Notably, these TNBC-bound STAT3 sites were further enriched near genes in pathways involved in cell migration and invasion, including extracellular matrix organization, extracellular structure organization, collagen metabolic process, anchoring junctions, adherens junctions and regulation of locomotion [23] (Supplementary Tables 2-3). These enriched pathways are related to invasion, and these findings point to a role for STAT3 in this key tumorigenic process in TNBCs.

\section{Comparison of STAT3 binding in cell lines with patient-derived tumor samples}

Because human breast cancer cell line models were used to characterize the genome-wide binding patterns of 
STAT3 in TNBC, we next determined if these genomewide binding patterns are also seen in primary tissue from TNBC tumors. We performed ChIP-seq on STAT3 in two frozen TNBC patient tumors [24], and identified 9,074 and 12,780 STAT3 binding events. We observed that STAT3 binding in TNBC tumor tissues is highly correlated with binding sites identified in basal TNBC cell lines (Figure 1). Similarly, STAT3 binding sites in tumors and cell lines are enriched for biological processes involving invasion mechanisms (Supplementary Figure 1). Importantly, these data indicate STAT3 cell line binding sites are also seen in vivo in patient tumors.

The high similarity of STAT3 binding patterns in TNBC tumors and cell lines led us to further analyze the binding patterns of STAT3 in two TNBC cell lines. Due to the heterogeneity of TNBC breast cancers, we selected two cell lines harboring broad molecular subtypes of TNBCbasal A (HCC70) and basal B (MDA-MB-231) tumors. ChIP-seq binding sites for each cell line was integrated with RNA-seq data to determine STAT3 driven gene regulation in TNBC. In HCC70, 20,808 high-confidence STAT3 binding sites were identified (Figure 1). Pairwise analysis of each ChIP-seq replicate revealed a significant correlation of binding sites across replicates (Spearman, rho=0.92) (Figure 1). These reproducible binding sites were significantly enriched for the canonical STAT3 motif (Figure 1D. Similarly, in the basal B cell line (MDAMB-231), there were 7,767 high-confidence binding sites in both replicates (Figure 1). Again, these binding sites had both a high correlation coefficient (Spearman, rho $=0.80)$, and a significant enrichment of the canonical STAT3 motif (Figure 1). The high-confidence binding sites for both cells lines were used for integration with RNAseq data to characterize the effects of STAT3 binding on gene expression.

\section{Effects of STAT3 on gene expression in TNBC cell lines}

To ascertain which genes are regulated by STAT3 in these basal TNBC cell lines, we performed RNAseq under control culture conditions and after 96 hour siRNA-mediated knockdown of STAT3 (Supplementary Figure 2) [25]. Analysis of the RNA-seq data revealed 737 differentially expressed transcripts in HCC70 (Figure 2 ) and 548 differentially expressed transcripts in MDA-

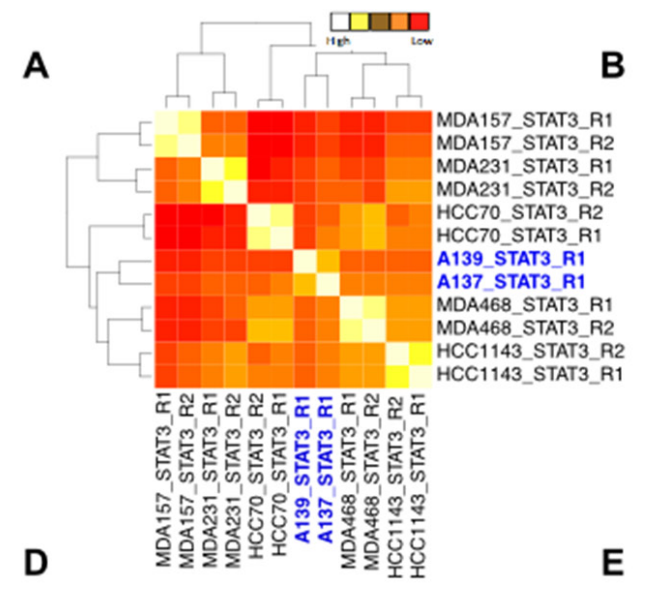

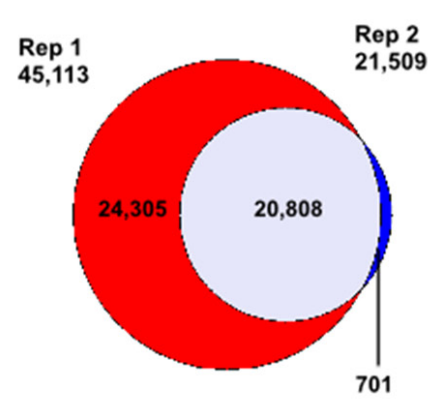

97\% Overlap of Called Peaks

E

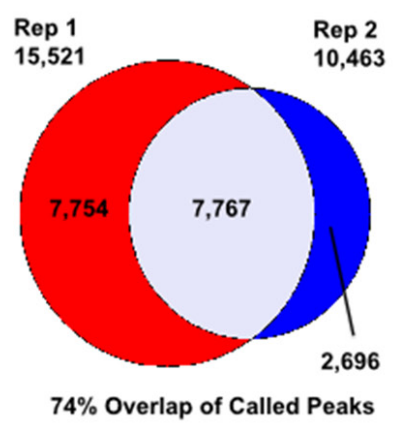

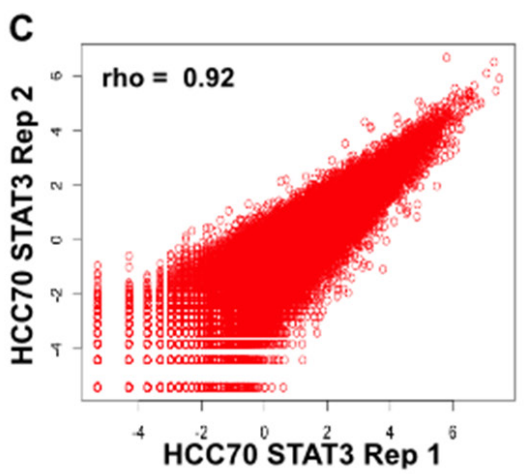

$\mathbf{F}$

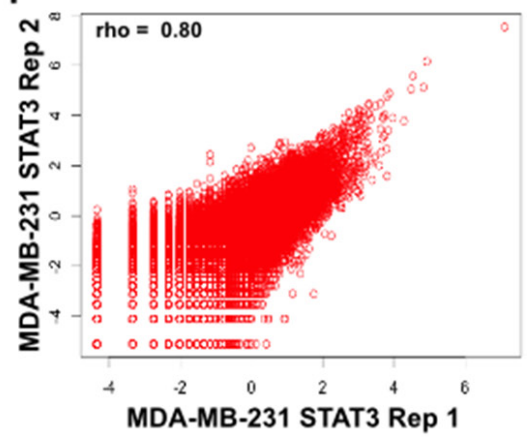

Figure 1: STAT3 binds in a subtype-specific manner across basal TNBC breast cancer. A. Heatmap of Spearman rank correlations between all pairwise comparisons of STAT3 binding sites across basal TNBC cell lines and TNBC tumors (blue). B. Venn diagram of replicate STAT3 binding sites in basal breast cancer cell line HCC70. 20,808 high-confidence binding sites were called in both replicates. C. Correlation plot of STAT3 binding sites in HCC70 replicates (Spearman rho=0.92) D. Canonical STAT3 motif enriched in binding sites in HCC70 and MDA-MB-231. E. Venn diagram of replicate STAT3 binding sites in basal breast cancer cell line MDAMB-231. 7,767 high-confidence binding sites were called in both replicates. F. Correlation plot of STAT3 binding sites in MDA-MB-231 replicates (Spearman, rho $=0.80$ ). 
MB-231 (Figure 2) (> 2.0-fold differences between siRNA treated cells and non-targeting vehicle controls).

Integration of the RNA-seq and STAT3 ChIPseq datasets in HCC70 cells showed that $50 \%$ of the differentially regulated transcripts (245 transcripts downregulated and 231 transcripts up-regulated; p-value < $2.22 \times 10^{-16}$ and $\mathrm{p}$-value $<5.64 \times 10^{-14}$, respectively, $\mathrm{k}$-s test) have a STAT3 binding site within $50 \mathrm{~kb}$ of their transcriptional start site (TSS) (Figure 2). These
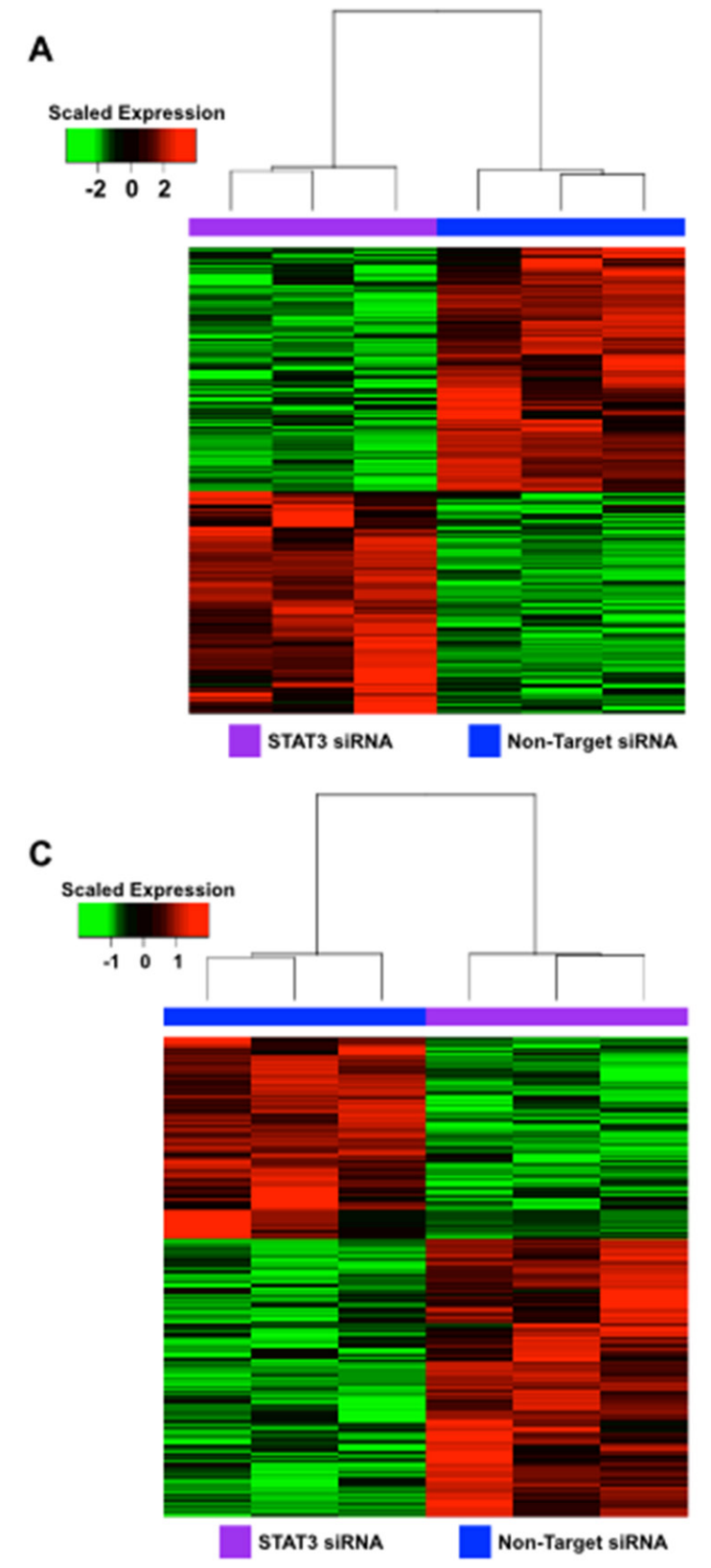

differentially expressed transcripts within $50 \mathrm{~kb}$ of a TSS are likely regulated by STAT3 in HCC70. Similarly in the MDA-MB-231 cell line, 216 genes (104 transcripts downregulated and 112 transcripts up-regulated; p-value $<8.07$ x $10^{-09}$ and $\mathrm{p}$-value $<1 \times 10^{-16}$, respectively, k-s test) were identified to have a STAT3 binding site within $50 \mathrm{~kb}$ of the gene's TSS (Figure 2). In each condition, STAT3 binding sites were significantly closer to differentially expressed gene TSSs than random transcripts, which is consistent

\section{B}

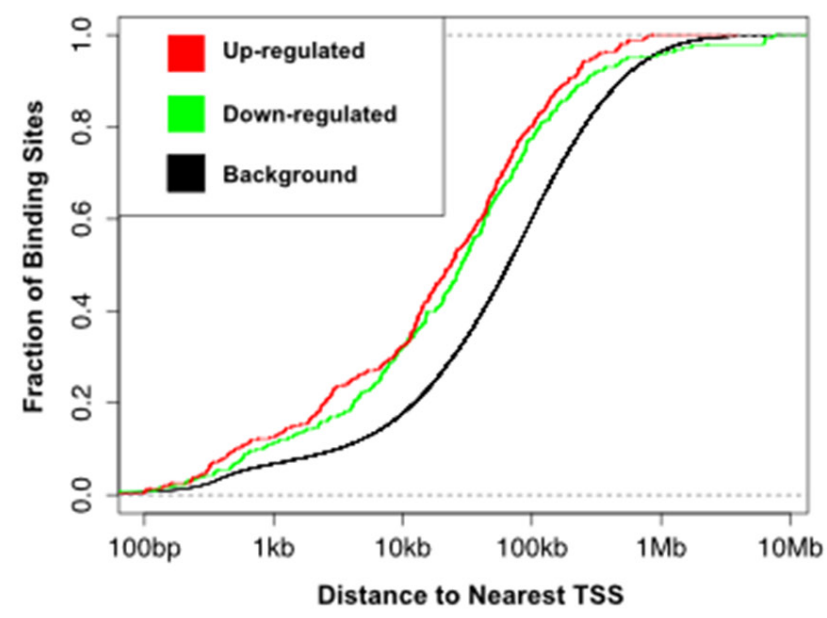

D

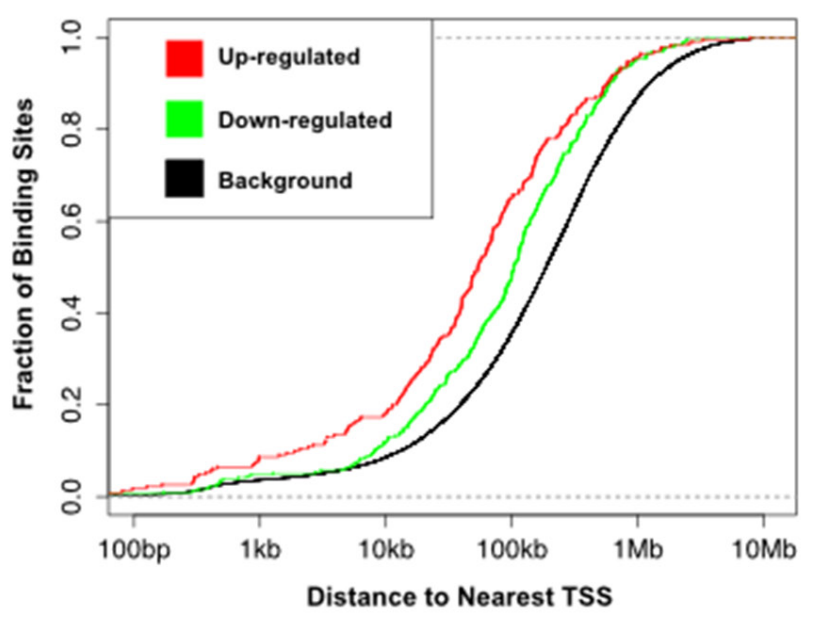

Figure 2: STAT3 binds and regulates genes in basal TNBC cell lines. A. Heatmap of 737 differentially expressed gene transcripts in HCC70 in response to STAT3 knockdown (>2.0-fold differences between siRNA treated cells and non-targeting vehicle controls) B. Cumulative distribution function plot of STAT3 binding sites near differentially expressed transcripts in HCC70. C. Heatmap of 548 differentially expressed gene transcripts in MDA-MB-231 in response to STAT3 knockdown ( $>2.0$-fold differences between siRNA treated cells and non-targeting vehicle controls) D. Cumulative distribution function plot of STAT3 binding sites near differentially expressed transcripts in MDA-MB-231. 
with regulation of gene expression. These findings suggest STAT3 acts more as a distal regulator in the context of TNBC, supporting previous findings of STAT3 distal gene regulation during normal development and in disease states [26].

\section{STAT3 regulates genes involved in cellular invasion and migration processes}

Discovery of STAT3 transcriptional activity in TNBC led us to assess the transcripts commonly regulated by this factor in both TNBC cell lines. We identified 22 common transcripts likely regulated by STAT3 (Supplementary Table 4). This small signature was not enriched for any biological processes; however, it provides insight to the potential regulatory mechanisms of STAT3 in TNBC. Interestingly, these findings indicate that STAT3 transcriptional activation in basal TNBC is heterogeneous, consistent with the heterogeneity of the phenotypes of TNBC in different patients. Therefore, we analyzed the direct target signatures unique to each cell line to discover pathways likely regulated by STAT3 in TNBC subtypes.

In the previously described HCC70 transcript signature, we discovered pathway enrichments activated by STAT3 (down-regulated after STAT3 knockdown) to include matrisome $\left(\mathrm{FDR}=7.85 \times 10^{-12}\right)$, inflammatory response $\left(\mathrm{FDR}=2.51 \times 10^{-04}\right)$, and immunity $\left(\mathrm{FDR}=1.26 \times 10^{-03}\right) \quad($ Table 1$)$. By contrast, pathway enrichment for transcripts likely repressed by STAT3 (up-regulated upon STAT3 knockdown) include matrisome $\left(\mathrm{FDR}=2.96 \times 10^{-04}\right)$, secreted factors $\left(\mathrm{FDR}=1.10 \times 10^{-07}\right)$, and epithelial to mesenchymal transition $(\mathrm{EMT})\left(\mathrm{FDR}=1.17 \times 10^{-05}\right)$ (Table 1). In MDAMB-231, pathway enrichments for transcripts likely activated by STAT3 included G-protein coupled receptor (GPCR) ligand binding $\left(\mathrm{FDR}=2.59 \times 10^{-04}\right)$, matrisome $\left(\mathrm{FDR}=8.54 \times 10^{-04}\right)$, and complement and coagulation cascades $\left(\mathrm{FDR}=8.59 \times 10^{-04}\right)$ (Table 2$)$. Similar to HCC70, STAT3 in MDA-MB-231 was found likely to repress pathways enriched for transcripts involving secreted factors $\left(\mathrm{FDR}=2.03 \times 10^{-06}\right)$, matrisome $\left(\mathrm{FDR}=9.18 \times 10^{-06}\right)$, and matrisome associated $\left(\mathrm{FDR}=2.64 \times 10^{-04}\right) \quad$ (Table 2). We also performed STAT3 ChIP-seq and RNA-seq (following STAT3 knockdown) in three additional cell lines (Supplementary Figures 2-4). Integration of these datasets reveals significant enrichments for invasion processes (Supplementary Tables 5-7). These results further confirm a key role for STAT3 as a regulator of invasion in TNBC. The enrichments are relevant because TNBC subtypes are likely to be invasive and metastatic, contributing to the aggressive nature of these cancers. STAT3 may play an important role in remodeling the extracellular matrix, exploiting inflammatory mechanisms, and evading immune surveillance to create a metastatic niche to facilitate spread of the cancer.

\section{Cellular invasion assays support a role for STAT3 in regulating invasion in TNBC}

Our results suggest a potential role for STAT3 in cellular invasion and migration in TNBC. We further investigated its role in invasion in HCC70 and MDAMB-231 cells by siRNA-mediated knockdown of STAT3 followed by matrigel transwell invasion assays. We observed a significant $44 \%($ t-test, $\mathrm{p}$-value $<0.0001)$ and $42 \%$ decrease (t-test, p-value $<0.0001$ ) in invasion of HCC70 and MDA-MB-231 cells, respectively transfected with the STAT3 siRNA pool (Figure 3). The significant reduction of invasive potential in both cell lines reveals a functional regulatory role for STAT3 in the invasive properties characteristic of TNBCs. To ensure that STAT3 knockdown did not simply result in reduced overall viability of the cells, we also performed cellular proliferation assays, but did not observe significant differences in the viability of HCC70 or MDA-MB-231 cells, even after 96 hours of STAT3 knockdown treatment (Figure 3). Collectively, these data support a unique role for STAT3 in cellular invasion and migration.

\section{DISCUSSION}

This work reveals the transcriptional program regulated by STAT3 in TNBC and its enrichment for invasion related processes. Invasion involves the intravasation of cancer cells from the primary tumor location into the vasculature; this is followed by cell metastasis to the distal tumor site [27-29]. It is well established that TNBC tumors are metastatic and chemoresistant in nature, which are contributing factors to the lack of targeted therapy for this breast cancer subtype. Various gene expression studies have characterized the genes expressed in basal TNBC, distinguishing this cancer from other breast cancer subtypes. These TNBC specific genes contribute to the aggressive biology of this cancer; however, the upstream transcriptional regulators remain elusive. To facilitate targeted therapy, the upstream transcriptional regulators of TNBC gene signatures must be identified.

Our studies revealed STAT3 as a key regulator of basal TNBC. STAT3 is constitutively activated in basal TNBCs; however, full characterization of its genomic regulatory mechanisms is undetermined. We conducted ChIP-seq to characterize the genome-wide binding patterns of STAT3 in TNBC tumors and cell lines. STAT3 binding in cell lines and tumors was enriched near pathways involving invasion, such as extracellular matrix remodeling and cell migration. From these results, we inferred that STAT3 appears to be involved in the regulation of invasion mechanisms in TNBC.

Integration of RNA-seq data from TNBC cell lines, after knockdown of STAT3, allowed for the prediction of 
Table 1: Top 10 GSEA enrichments of differentially expressed genes in response to 96 hour STAT3 knockdown in HCC70

\begin{tabular}{|c|c|}
\hline $\begin{array}{l}\text { Signatures Directly Activated by STAT3 in HCC70 } \\
\text { (Down-regulated Upon STAT3 Knockdown) }\end{array}$ & FDR q-value \\
\hline NABA_MATRISOME & $7.85 \mathrm{E}-12$ \\
\hline NABA_MATRISOME_ASSOCIATED & $1.46 \mathrm{E}-06$ \\
\hline HALLMARK_INFLAMMATORY_RESPONSE & $2.51 \mathrm{E}-04$ \\
\hline NABA_CORE_MATRISOME & $2.51 \mathrm{E}-04$ \\
\hline NABA_ECM_REGULATORS & $6.92 \mathrm{E}-04$ \\
\hline $\begin{array}{l}\text { KEGG_COMPLEMENT_AND_COAGULATION_ } \\
\text { CASCADES }\end{array}$ & $1.17 \mathrm{E}-03$ \\
\hline REACTOME_IMMUNE_SYSTEM & $1.26 \mathrm{E}-03$ \\
\hline NABA_ECM_GLYCOPROTEINS & $1.26 \mathrm{E}-03$ \\
\hline $\begin{array}{l}\text { REACTOME_REGULATION_OF_COMPLEMENT_ } \\
\text { CASCADE }\end{array}$ & $2.42 \mathrm{E}-03$ \\
\hline BIOCARTA_IL22BP_PATHWAY & $3.33 \mathrm{E}-03$ \\
\hline $\begin{array}{l}\text { Signatures Directly Repressed by STAT3 in HCC70 (Up- } \\
\text { regulated Upon STAT3 Knockdown) }\end{array}$ & FDR q-value \\
\hline NABA_MATRISOME & $2.96 \mathrm{E}-14$ \\
\hline NABA_MATRISOME_ASSOCIATED & $1.30 \mathrm{E}-10$ \\
\hline NABA_SECRETED_FACTORS & $1.10 \mathrm{E}-07$ \\
\hline $\begin{array}{l}\text { HALLMARK_EPITHELIAL_MESENCHYMAL_ } \\
\text { TRANSITION }\end{array}$ & $1.17 \mathrm{E}-05$ \\
\hline $\begin{array}{l}\text { REACTOME_CLASS_A1_RHODOPSIN_LIKE_ } \\
\text { RECEPTORS }\end{array}$ & 3.13E-05 \\
\hline REACTOME_GPCR_LIGAND_BINDING & 4.27E-05 \\
\hline $\begin{array}{l}\text { REACTOME_PEPTIDE_LIGAND_BINDING_ } \\
\text { RECEPTORS }\end{array}$ & $6.05 \mathrm{E}-05$ \\
\hline HALLMARK_COAGULATION & $9.12 \mathrm{E}-05$ \\
\hline HALLMARK_INFLAMMATORY_RESPONSE & $8.62 \mathrm{E}-04$ \\
\hline HALLMARK_TNFA_SIGNALING_VIA_NFKB & 8.62E-04 \\
\hline
\end{tabular}

Enrichments represent hallmark and canonical pathways in GSEA database. STAT3 responsive genes are enriched for processes involving invasion

genes likely regulated by STAT3. We found that genes potentially regulated by STAT3 were mostly cell line specific, further corroborating the known heterogeneity of basal TNBC. Despite little overlap of genes likely regulated by STAT3 in the cell lines, the most significantly enriched process in both cell lines was for matrisome (the ensemble of extracellular matrix (ECM) proteins and related ECM proteins) formation [30, 31]. Matrisome processes were activated and repressed by STAT3, and it is likely that STAT3 promotes the degradation of the
ECM and inhibits its maintenance to promote invasion. The TNBC matrisome was indeed impacted by STAT3 perturbation, as the transwell assays allowed us to functionally test regulation of the matrisome by STAT3. Knockdown of STAT3 resulted in decreased invasive potential of the basal TNBC cell lines across matrigelcoated wells. Taken together, STAT3 regulates proinvasion and pro-metastatic gene signatures in a TNBC subtype specific manner. 
Table 2: Top 10 GSEA enrichments of differentially expressed genes in response to 96 hour STAT3 knockdown in MDA-MB-231

\begin{tabular}{|c|c|}
\hline $\begin{array}{l}\text { Signatures Directly Activated by STAT3 in MDA- } \\
\text { MB-231 (Down-regulated Upon STAT3 Knockdown) }\end{array}$ & FDR q-value \\
\hline REACTOME_GPCR_LIGAND_BINDING & $2.59 \mathrm{E}-04$ \\
\hline $\begin{array}{l}\text { REACTOME_CLASS_A1_RHODOPSIN_LIKE_ } \\
\text { RECEPTORS }\end{array}$ & 2.77E-04 \\
\hline BIOCARTA_CLASSIC_PATHWAY & $5.36 \mathrm{E}-04$ \\
\hline BIOCARTA_COMP_PATHWAY & 8.54E-04 \\
\hline NABA_MATRISOME & 8.54E-04 \\
\hline $\begin{array}{l}\text { KEGG_COMPLEMENT_AND_COAGULATION_ } \\
\text { CASCADES }\end{array}$ & 8.59E-04 \\
\hline KEGG_HEMATOPOIETIC_CELL_LINEAGE & $1.94 \mathrm{E}-03$ \\
\hline REACTOME_G_ALPHA_WE_SIGNALLING_EVENTS & $2.03 \mathrm{E}-03$ \\
\hline REACTOME_GPCR_DOWNSTREAM_SIGNALING & 4.23E-03 \\
\hline NABA_ECM_REGULATORS & 4.23E-03 \\
\hline $\begin{array}{l}\text { Signatures Directly Repressed by STAT3 in MDA- } \\
\text { MB-231 (Up-regulated Upon STAT3 Knockdown) }\end{array}$ & FDR q-value \\
\hline NABA_SECRETED_FACTORS & $2.03 \mathrm{E}-06$ \\
\hline HALLMARK_HYPOXIA & $9.18 \mathrm{E}-06$ \\
\hline NABA_MATRISOME & $9.18 \mathrm{E}-06$ \\
\hline $\begin{array}{l}\text { KEGG_CYTOKINE_CYTOKINE_RECEPTOR_ } \\
\text { INTERACTION }\end{array}$ & $3.72 \mathrm{E}-05$ \\
\hline HALLMARK_TNFA_SIGNALING_VIA_NFKB & $1.16 \mathrm{E}-04$ \\
\hline NABA_MATRISOME_ASSOCIATED & $2.63 \mathrm{E}-04$ \\
\hline REACTOME_ACTIVATION_OF_GENES_BY_ATF4 & $1.35 \mathrm{E}-03$ \\
\hline $\begin{array}{l}\text { REACTOME_PERK_REGULATED_GENE_ } \\
\text { EXPRESSION }\end{array}$ & $1.52 \mathrm{E}-03$ \\
\hline HALLMARK_INFLAMMATORY_RESPONSE & $1.52 \mathrm{E}-03$ \\
\hline HALLMARK_UNFOLDED_PROTEIN_RESPONSE & $2.88 \mathrm{E}-03$ \\
\hline
\end{tabular}

Enrichments represent hallmark and canonical pathways in GSEA database. STAT3 responsive genes are enriched for processes involving invasion

STAT3 has been implicated in various cancers, including breast, ovarian, prostate, and lung cancers, as having a regulatory role in cancer development, including transformation, proliferation, EMT, invasion, and metastasis [16]. However, until now, the role of STAT3 in the context of basal TNBC was unknown. Recent studies revealed that phosphorylated STAT3 is associated with genes involved in immunity, inflammation, and invasion in TNBC transcriptomic data from TCGA [32]. These findings further supported a key regulatory role for STAT3 in TNBC. However, it did not describe direct gene signatures regulated by STAT3, nor functional characterization of the role of STAT3 in invasion in TNBC. Our work builds on this study as we further uncovered gene signatures likely regulated by STAT3 that corroborates the functional enrichments described by independent studies [32]. Moreover, we provide functional data to support a role for STAT3 in the regulation of cellular invasion processes in TNBC. While the direct gene targets of STAT3 are subtype specific across TNBC, 
indicative of the heterogeneity of this disease, our findings suggest this factor plays a universal role of regulating invasion in this cancer.

Further investigation must be conducted to unravel the role of STAT3 in actual metastasis of TNBC tumor cells to a secondary site. Development of TNBC xenograft mouse models followed by STAT3 perturbation will assist in discovering if STAT3 is indeed a therapeutic target for inhibiting metastasis of TNBC tumors. Direct inhibition of STAT3 may potentially improve chemotherapy response and decrease metastasis in TNBC, ultimately improving overall survival of TNBC patients. STAT3 subtype specific gene signatures could serve as potential biomarkers for determining the specific subtype of TNBC tumor and appropriate choice of STAT3 inhibition and chemotherapy for personalized treatment. Additional studies aimed at
A.

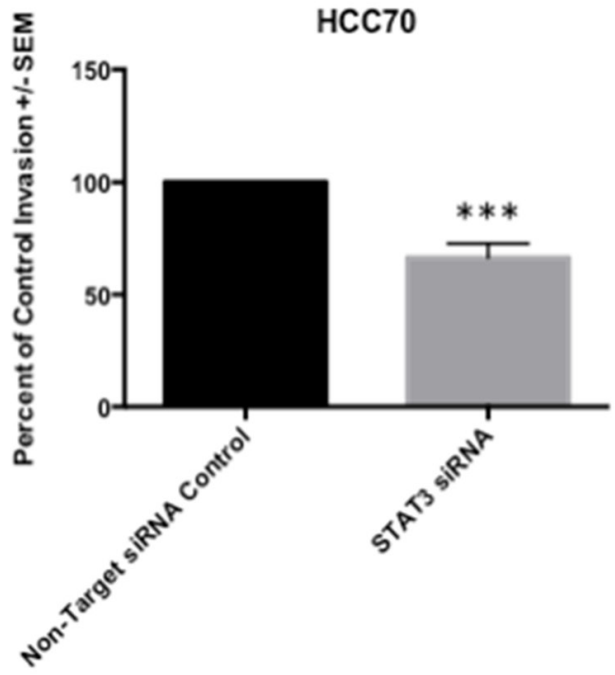

C.

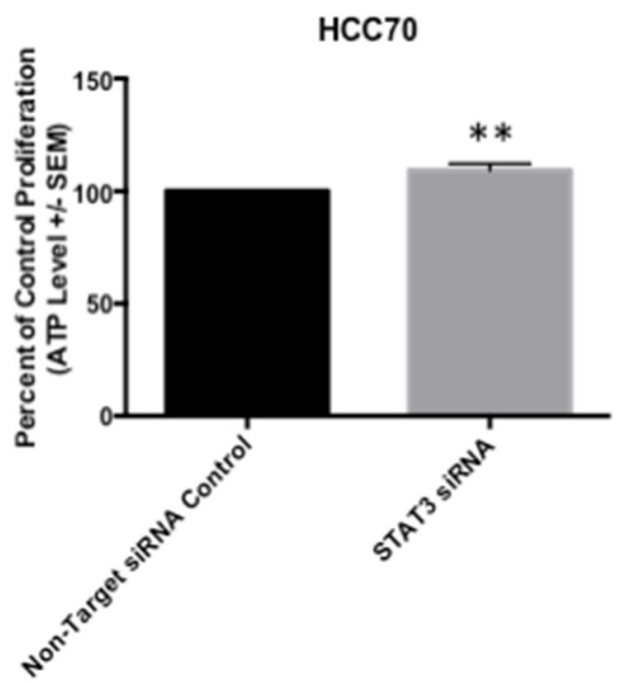

B.

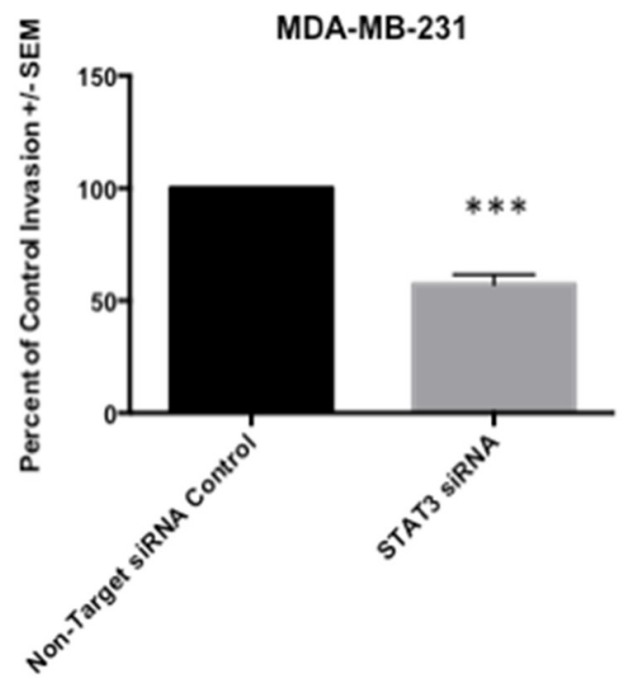

D.

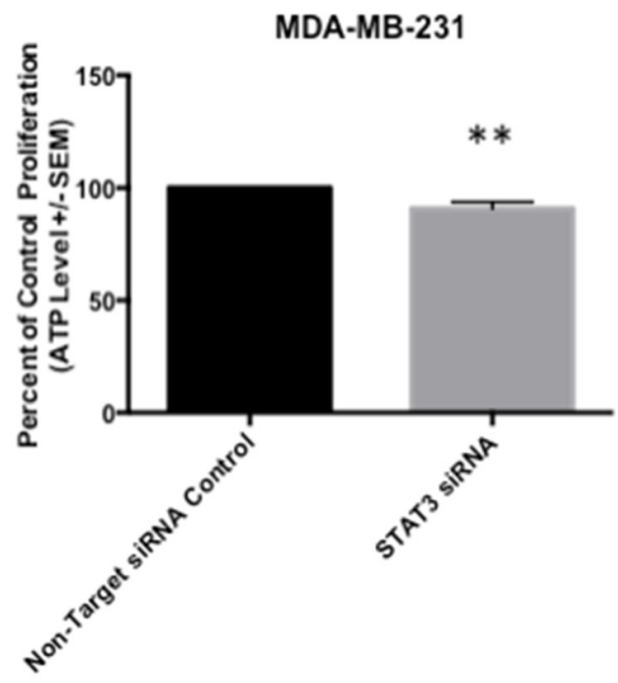

Figure 3: STAT3 regulates a gene signature associated with an invasion phenotype in basal TNBC. (Data represents mean +/- SEM) A. Transwell invasion after STAT3 knockdown in HCC70 resulted in 1.6-fold reduced invasion (t-test, p-value $<0.0001)$. B. Transwell invasion after STAT3 knockdown in MDA-MB-231 resulted in 1.9-fold reduced invasion (t-test, p-value $<0.0001$ ). C. HCC70 proliferation measure after siRNA mediated STAT3 knockdown for 96 hrs. Knockdown of STAT3 did not result in reduced proliferation, but increased proliferation by $10 \%$ (t-test, p-value $<0.0037$ ). D. MDA-MB-231 proliferation measure after STAT3 knockdown for $96 \mathrm{hrs}$. Knockdown of STAT3 resulted in $10 \%$ decrease in proliferation (t-test, p-value $<0.0040$ ). 
understanding the pharmacological response of the TNBC subtype specific STAT3 targets are needed to develop personalized therapies for inhibiting this factor.

\section{MATERIALS AND METHODS}

\section{Cell culture and harvest}

The following cell lines: MDA-MB-231, MDAMB-157, MDA-MB-468 were cultured in DMEM media (Gibco, ThermoFisher Scientific, Waltham, MA) supplemented with 10\% fetal bovine serum (Hyclone, GE Healthcare, Logan, UT), $1 \mathrm{mM}$ sodium pyruvate, and nonessential amino acids (Gibco). HCC70 and HCC1143 were cultured in RPMI media (Gibco), supplemented with 10\% fetal bovine serum (Hyclone), 10 mM HEPES (SigmaAldrich, St. Louis, MO), $1 \mathrm{mM}$ sodium pyruvate (Gibco), and $4.5 \mathrm{~g} / 1$ glucose (Sigma-Aldrich). Cells were passaged and harvested at $80 \%$ confluence. Prior to harvest, cells were treated for $72 \mathrm{hr}$ or $96 \mathrm{hr}$ with either $10 \mu \mathrm{M}$ STAT3 siRNA or an equal concentration of control non-targeting SiRNA.

For ChIP-seq experiments, protein-DNA complexes were covalently cross-linked by incubating cells in $1 \%$ formaldehyde for 10 minutes at room temperature. Cells were incubated with $0.125 \mathrm{M}$ glycine for 5 minutes, to quench cross-linking reaction. Cells were washed and scraped with PBS (pH 7.4) (Lonza, Walkersville, MD). Cells were lysed with Farnham Lysis Buffer (5mM PIPES at $\mathrm{pH} 8.0,85 \mathrm{mM} \mathrm{KCl}, 0.5 \% \mathrm{NP}-40)$ containing protease inhibitor (Roche Diagnostics, Indianapolis, IN). Cell lysate was centrifuged at $2000 \mathrm{rpm}$ for 5 minutes at $4^{\circ} \mathrm{C}$. The crude nuclear extract contained in the supernatant was stored at $-80^{\circ} \mathrm{C}$.

For RNA-seq, cells were treated with Buffer RL (Norgen Biotek Corporation, Thorold, ON, CAN) and stored at $-80^{\circ} \mathrm{C}$. Cell lysate was collected after addition of $100 \%$ molecular biology grade ethanol. Total RNA was extracted using the Norgen Animal Tissue RNA Purification Kit.

\section{RNAi knockdown of STAT3}

We used an ON-TARGETplus Human STAT3 siRNA kit from GEHealthcare (L-003544-00-0005, GE Healthcare, Logan, UT). This SMARTpool siRNA contains four pooled siRNAs, each targeting a separate region of the STAT3 RNA sequence. We also used ONTARGETplus Non-targeting siRNA \#1 (D-001810-01-05) as a non-targeting control. The siRNA SMARTpool and Non-targeting siRNA target sequences are below:

ON-TARGETplus SMARTpool siRNA J-00354407, STAT3

Target Sequence: GAGAUUGACCAGCAGUAUA

ON-TARGETplus SMARTpool siRNA J-00354408, STAT3
Target Sequence: CAACAUGUCAUUUGCUGAA ON-TARGETplus SMARTpool siRNA J-00354409, STAT3

Target Sequence: CCAACAAUCCCAAGAAUGU ON-TARGETplus SMARTpool siRNA J-00354410, STAT3

Target Sequence: CAACAGAUUGCCUGCAUUG ON-TARGETplus Non-targeting siRNA \#1

Target Sequence: UGGUUUACAUGUCGACUAA

The siRNA transfection experiments were performed in 6-well and 96-well cell culture plates in triplicate and quintuplicate, respectively, and included the non-targeting control. For transfection, the Lipofectamine RNAiMAX Transfection Reagent (ThermoFisher Scientific) was used. Lipofectamine and siRNAs were prepared per manufacturer's instructions (ThermoFisher Scientific). To each well containing cells, in the 96-well plate experiments (viability), we added $10 \mathrm{ul}$ of the siRNA-transfection reagent mix diluted in Opti-MEM I Reduced Serum Medium (ThermoFisher Scientific); this resulted in 1 pmol siRNA in $0.3 \mu$ Lipofectamine RNAiMAX reagent per well. For 6-well cell culture plate experiments, $250 \mu \mathrm{l}$ siRNA-transfection reagent mix was added to each well, for a final concentration of $25 \mathrm{pmol}$ siRNA in $7.5 \mu \mathrm{l}$ Lipofectamine RNAiMAX reagent per well.

\section{SDS-PAGE and western blotting}

To confirm knockdown of STAT3 protein levels, SDS-PAGE followed by western blotting was performed. HCC70 and MDA-MB-231 cells were seeded in sixwell plates, at a density of 300,000 (MDA-MB-231) or 600,000 (HCC70) cells per well. After 96 hours, cells were harvested via trypsinization followed by centrifugation at 2,000 rpm for 5 minutes. Cell pellets were washed in $1 \mathrm{~mL}$ PBS, centrifuged a second time, and stored at $-80^{\circ} \mathrm{C}$ until immunoprecipitation step. Cell pellets were resuspended in $100 \mu \mathrm{l}$ RIPA buffer containing protease inhibitors, and incubated, on ice, for 30 minutes. Cells were dounced with a syringe to further lyse cells, followed by centrifugation at $14,000 \mathrm{rpm}$ for 10 minutes. The supernatant was collected and protein concentrations were measured using the Qubit Protein Assay kit and Qubit fluorometer (ThermoFisher Scientific). $40 \mu \mathrm{g}$ protein for each cell line and condition were mixed with an equal volume of Laemelli Buffer (Bio-Rad, Hercules, CA) supplemented with 10\% Beta-mercaptoethanol (Sigma). Protein samples were heated in a $95-100^{\circ} \mathrm{C}$ water bath for ten minutes. Protein lysate samples and SeeBlue Plus2 (ThermoFisher Scientific) and Magic Mark XP (ThermoFisher Scientific) protein standards were loaded onto a pre-cast SDSpolyacrylamide gel (Bio-Rad) assembled in a reservoir containing 1X Tris/Glycine buffer (BioRad).

The SDS-PAGE gel was run for 1.5 hours at 150V. Proteins were transferred to a nitrocellulose 
membrane using the iBlot gel transfer device, per the manufacturer's instructions, using program 3 for seven minutes (ThermoFisher Scientific). Following transfer, the protein-containing membrane was placed in blocking reagent (PBS $+\mathrm{NF}$ dairy milk $+10 \%$ Tween), followed by shaking for 1 hour at room temperature. Blocking reagent was discarded and wash buffer containing antibodies targeting STAT3 (Santa-Cruz) phosphorylated STAT3 (Ser727) (Cell Signaling Technology), $\beta$-actin (loading control) (Cell Signaling Technology) were added to the membrane and incubated overnight at $4^{\circ} \mathrm{C}$, with rotation. The membrane was washed three times, for ten minutes each, with western wash buffer (1X PBS with $0.05 \%$ Tween 20). Wash buffer containing anti-rabbit or antimouse secondary antibody was added to the membrane and incubated for 1 hour at room temperature. The membrane was again, washed three times for ten minutes. To visualize blotted proteins, SuperSignal West Femto working solution was prepared by mixing equal parts of stable peroxide and luminol/enhancer, per manufacturer's instructions (ThermoFisher Scientific) and incubated with the membrane for 5 minutes. Imaging was conducted using the UVP imaging system.

\section{Matrigel invasion assays}

To determine if STAT3 regulates invasion, 300,000 (HCC70) or 75,000 (MDA-MB-231) cells were seeded into six-well plates and transfected with STAT3 siRNAs and non-targeting siRNAs as described above. Cells were incubated for 72 hours (HCC70) or 96 hours (MDA$\mathrm{MB}-231)$ at $37^{\circ} \mathrm{C} / 5 \% \mathrm{CO}_{2}$. Cells were harvested via trypsinization followed by centrifugation at 2,000 rpm for five minutes. Cell pellets were resuspended in $10 \mathrm{ml}$ media and quantified using a hemocytometer. Cells were diluted to a concentration of 40,000 cells $/ \mathrm{ml}$ in serum free media and seeded onto Corning Biocoat matrigel invasion chambers (Corning, Bedford, MA) as previously described [33]. Student's t-test was applied to calculate significance using GraphPad Prism (GraphPad Software, La Jolla, CA).

\section{Cell viability assays}

Cell viability assays were conducted in 96-well cell culture plates. HCC70 and MDA-MB-231 cells were transfected with a siRNA targeting STAT3 or a non-targeting siRNA vehicle control for 96 hours. Cell proliferation was measured using the ATPLite Assay (Perkin Elmer, Waltham, MA) for cell metabolism.

\section{ChIP-seq}

To characterize genome-wide binding patterns of STAT3 in TNBC cell lines, ChIP-seq was performed as previously described [34]. Antibodies for STAT3 (Santa Cruz, sc-482, Santa Cruz Biotechnology, Santa Cruz, CA) were used. Replicate experiments with high similarity (>
$80 \%$ concordance across called peaks), were combined into a single data set of high confidence binding sites.

Measurement of STAT3 binding in TNBC tumor tissues was performed as previously described [24].

\section{RNA-seq}

Cells were lysed with Buffer RL (Norgen) containing $10 \%$ beta-mercaptoethanol. Cell lysate was collected after addition of $300 \mu 1$ 100\% molecular biology grade ethanol. Total RNA was extracted using the Animal Tissue RNA Purification Kit (Norgen). RNA-seq libraries were prepared from $250 \mathrm{ng}$ total RNA via polyA-selection (Dynabead mRNA Purification Kit, ThermoFisher Scientific) followed by transposase-mediated non-stranded library construction [25]. Each experimental treatment was performed in triplicate. Libraries were pooled and sequenced on an Illumina HiSeq 2000 sequencer using paired-end $50 \mathrm{bp}$ reads with a $6 \mathrm{bp}$ index read (Illumina, Inc., San Diego, CA). Pooled sequencing resulted in 26.5 million reads per library. Differential expression was measured using the DESeq2 program [35]. TopHat (version 1.4.1) was used to align RNA-seq paired reads to GENCODE (version 9.0) [36, 37]. Cufflinks (version 1.3.0) and BEDTools [38, 39] were used to calculate raw counts for each GENCODE transcript. For this study X and $\mathrm{Y}$ chromosome transcripts were omitted.

\section{Integrated genomic analysis}

For each ChIP-seq replicate, peaks were identified and reported using the MACS peak caller program [40]. Motif analysis for binding sites identified in individual replicates was performed using MEME [41]. After identification of STAT3 binding sites, the normalized read depth for each experiment was calculated by tabulating the number of reads across binding sites and normalizing the reads to the total number of aligned reads generated for each experiment. The normalized reads were mapped by centering each ChIP-seq peak summit around 100bp sequences. Spearman correlation coefficients were calculated across normalized STAT3 binding sites and clustered to identify differential STAT3 binding patterns across basal tumors and cell lines. STAT3 peaks common in TNBC cell lines and tumors were analyzed for gene ontology enrichment using the GREAT program [23]. In HCC70 and MDA-MB-231 ChIP-seq studies, only reproducible binding sites identified in replicates, were called as high-confidence bindings sites and used for integrated analysis with RNA-seq data sets.

Differential gene expression analysis was performed in the statistical software, $\mathrm{R}$ (version 3.2.1) [42], using DESeq2 (version 1.8.1), using the default settings [35]. Differentially expressed transcripts were filtered for only those transcripts with $>2.0$ fold differences between STAT3 siRNA treated cells and non-targeting siRNA 
vehicle controls. Differentially expressed transcripts were normalized, followed by hierarchical clustering using the hclust command in R (method="ward", distance="euclidean"). Differential transcripts were integrated with high confidence ChIP-seq binding sites and the central distribution function was calculated to predict what fraction of significant transcripts have a STAT3 binding site within $50 \mathrm{~kb}$ of the TSS. $50 \mathrm{~kb}$ was chosen as a cutoff based on data suggesting that the correlation between TF binding and gene expression tails off beyond about $100 \mathrm{~kb}$ [43]. The Komolgorov-Smirov test (KS-test) was implemented to determine the significant enrichment of STAT3 binding near differentially expressed transcripts compared to the background genome using the ks.test command in $\mathrm{R}$.

Differentially expressed genes with a nearby binding site were considered to be direct targets and were used for Hallmark and Canonical Pathway enrichments using Gene Set Enrichment Analysis [44, 45]. Student's t-tests were used to calculate significance of cell viability and invasion assays.

All ChIP-seq and RNA-seq datasets have been deposited in GEO (accession number: GSE85579).

\section{Abbreviations}

ChIP-seq: Chromatin Immunoprecipitation followed by massively parallel sequencing; ECM: Extracellular Matrix; ER: Estrogen Receptor; HER2: Human Epidermal Growth Factor Receptor 2; PR: Progesterone Receptor; STAT3: Signal Transducer and Activator of Transcription 3; TF(s): Transcription Factor(s); TNBC: Triple Negative Breast Cancer.

\section{ACKNOWLEDGMENTS}

We thank the members of the HudsonAlpha Genomic Services Laboratory for providing sequencing of ChIP and RNA libraries for this project, and members of the Myers lab for helpful comments and discussions.

\section{CONFLICTS OF INTEREST}

The authors declare no conflicts of interest.

\section{FUNDING}

This research was supported by funding from the HudsonAlpha Institute, Susan G. Komen (to AF), the Breast Cancer Research Foundation (to AF) and the NIH-National Cancer Institute Comprehensive Cancer Center Core Support Grant (5P30CA013148). JMM was supported by a fellowship from the National Science Foundation Graduate Research Fellowship
Program (NSF-GRFP). RMM and BNL were supported by a Cancer Research Fund from the state of Alabama. RCR was funded by the UAB MSTP grant (NIHMIGMS 5T32GM008361-21), SJC is supported by the HudsonAlpha's Tie the Ribbon Fund for Breast and Ovarian Cancer Research, and BNL was funded by the William J. Maier III Fellowship in Cancer Prevention (Prevent Cancer Foundation).

\section{Author contributions}

Conception and design: JMM, KEV, JG, BSR, DSS, LAS, DJB and RMM

Acquisition of data: JMM, KEV, JG, BSR, DSS, KMN, SKB, LAS, ECP, ALJ, BB, SEL and RMM

Analysis and interpretation of data: JMM, DSS, BSR, RCR, SKB, LAS, SJC and RMM

Writing, review and/or revision of manuscript: JMM, DSS, BNL, MKK, RCR, LAS, DJB and RMM

Administrative, technical or material support: PGO, $\mathrm{KCS}, \mathrm{AF}$, and WEG

\section{REFERENCES}

1. Desantis C, Ma J, Bryan L, Jemal A. Breast Cancer Statistics, 2013. 2013; 00: 1-11. doi: 10.3322/caac.21203.

2. Siegel RL, Miller KD, Jemal A. Cancer Statistics, 2016. 2016; 66: 7-30. doi: 10.3322/caac.21332.

3. Sorlie T, Perou CM, Tibshirani R, Aas T, Geisler S, Johnsen H, Hastie T, Eisen MB, van de Rijn M, Jeffrey SS, Thorsen T, Quist H, Matese JC, et al. Gene expression patterns of breast carcinomas distinguish tumor subclasses with clinical implications. Proc Natl Acad Sci U S A. 2001; 98: 10869 74. doi: 10.1073/pnas.191367098.

4. Zhang MH, Man HT, Zhao XD, Dong N, Ma SL. Estrogen receptor-positive breast cancer molecular signatures and therapeutic potentials (Review). Biomed reports. 2014; 2: 41-52. doi: 10.3892/br.2013.187.

5. Hoch RV, Thompson DA, Baker RJ, Weigel RJ. GATA-3 is expressed in association with estrogen receptor in breast cancer. Int J Cancer. 1999; 84: 122-8. doi: 10.1002/(SICI)1097-0215(19990420)84:2<122::AIDIJC5>3.0.CO;2-S.

6. Rastelli F, Crispino S. Factors predictive of response to hormone therapy in breast cancer. Tumori. 2008; 94 : 370-83.

7. Dent S, Oyan B, Honig A, Mano M, Howell S. HER2targeted therapy in breast cancer: a systematic review of neoadjuvant trials. Cancer Treat Rev. 2013; 39: 622-31. doi: 10.1016/j.ctrv.2013.01.002.

8. Tomao F, Papa A, Zaccarelli E, Rossi L, Caruso D, Minozzi M, Vici P, Frati L, Tomao S. Triple-negative breast cancer: New perspectives for targeted therapies. Onco Targets Ther. 2015; 8: 177-93. doi: 10.2147/OTT.S67673. 
9. Carey LA, Dees EC, Sawyer L, Gatti L, Moore DT, Collichio F, Ollila DW, Sartor CI, Graham ML, Perou CM. The triple negative paradox: primary tumor chemosensitivity of breast cancer subtypes. Clin Cancer Res. 2007; 13: 2329-34. doi: 10.1158/1078-0432. CCR-06-1109.

10. Hudis CA, Gianni L. Triple-negative breast cancer: an unmet medical need. Oncologist. 2011; 16: 1-11. doi: 10.1634/theoncologist.2011-S1-01.

11. Anders CK, Carey LA. Biology, metastatic patterns, and treatment of patients with triple-negative breast cancer. Clin Breast Cancer. 2009; 9: S73-81. doi: 10.3816/ CBC.2009.s.008.

12. Dent R, Hanna WM, Trudeau M, Rawlinson E, Sun P, Narod SA. Pattern of metastatic spread in triple-negative breast cancer. Breast Cancer Res Treat. 2009; 115: 423-8. doi: 10.1007/s10549-008-0086-2.

13. Lehmann BD, Bauer JA, Chen $\mathrm{X}$, Sanders ME, Chakravarthy AB, Shyr Y, Pietenpol JA. Identification of human triple-negative breast cancer subtypes and preclinical models for selection of targeted therapies. J Clin Invest. 2011; 121: 2750. doi: 10.1172/JCI45014DS1.

14. Perou CM, Sørlie T, Eisen MB, van de Rijn M, Jeffrey SS, Rees CA, Pollack JR, Ross DT, Johnsen H, Akslen LA, Fluge O, Pergamenschikov A, Williams C, et al. Molecular portraits of human breast tumours. Nature. 2000; 406: 74752. doi: 10.1038/35021093.

15. Deng X, Wang S, Deng A, Liu B, Edgerton SM, Lind SE, Wahdan R, Thor AD. Metformin targets Stat3 to inhibit cell growth and induce apoptosis in triple-negative breast cancers. Cell Cycle. 2012; 11: 335-42. doi: 10.4161/ cc.11.2.18813.

16. Xiong A, Yang Z, Shen Y, Zhou J, Shen Q. Transcription factor STAT3 as a novel molecular target for cancer prevention. Cancers (Basel). 2014; 6: 926-57. doi: 10.3390/ cancers6020926.

17. Walker SR, Nelson EA, Zou L, Chaudhury M, Signoretti S, Richardson A, Frank DA. Reciprocal effects of STAT5 and STAT3 in breast cancer. Mol Cancer Res. 2009; 7: 966-76. doi: 10.1158/1541-7786.MCR-08-0238.

18. Marotta LLC, Almendro V, Marusyk A, Shipitsin M, Schemme J, Walker SR, Bloushtain-qimron N, Kim JJ, Choudhury SA, Maruyama R, Wu Z, Gönen M, Mulvey LA, et al. The JAK2 / STAT3 signaling pathway is required for growth of CD44 + CD24 - stem cell - like breast cancer cells in human tumors. J Clin Invest. 2011; 121: 2723-35. doi: 10.1172/JCI44745DS1.

19. Carpenter RL, Lo HW. STAT3 target genes relevant to human cancers. Cancers (Basel). 2014; 6: 897-925. doi: 10.3390/cancers6020897.

20. Kamran MZ, Patil P, Gude RP. Role of STAT3 in cancer metastasis and translational advances. Biomed Res Int. 2013; 2013: 421821. doi: 10.1155/2013/421821.
21. Thakur R, Trivedi R, Rastogi N, Singh M, Mishra DP. Inhibition of STAT3, FAK and Src mediated signaling reduces cancer stem cell load, tumorigenic potential and metastasis in breast cancer. Sci Rep. 2015; 5: 10194. doi: 10.1038/srep10194.

22. Johnson DS, Mortazavi A, Myers RM, Wold B. Genomewide mapping of in vivo protein-DNA interactions. Science. 2007; 316: 1497-502. doi: 10.1126/science.1141319.

23. McLean CY, Bristor D, Hiller M, Clarke SL, Schaar BT, Lowe CB, Wenger AM, Bejerano G. GREAT improves functional interpretation of cis-regulatory regions. Nat Biotechnol. 2010; 28: 495-501. doi: 10.1038/nbt.1630.

24. Savic D, Gertz J, Jain P, Cooper GM, Myers RM. Mapping genome-wide transcription factor binding sites in frozen tissues. Epigenetics Chromatin. 2013; 6: 30. doi: 10.1186/1756-8935-6-30.

25. Gertz J, Varley KE, Davis NS, Baas BJ, Goryshin IY, Vaidyanathan R, Kuersten S, Myers RM. Transposase mediated construction of RNA-seq libraries. Genome Res. 2012; 22: 134-41. doi: 10.1101/gr.127373.111.

26. Zhao Y, Zeng C, Tarasova NI, Chasovskikh S, Dritschilo A, Timofeeva OA. A new role for STAT3 as a regulator of chromatin topology. Transcription. 2013; 4: 227-31. doi: $10.4161 /$ trns.27368.

27. Hanahan, D, Weinberg, RA. The Hallmarks of Cancer. Cell. 2000; 100:57-70. doi: http://dx.doi.org/10.1016/j. cell.2011.02.013.

28. Hanahan D, Weinberg RA. Hallmarks of cancer: the next generation. Cell. 2011; 144: 646-74. doi: 10.1016/j. cell.2011.02.013.

29. van Zijl, F, Krupitza, G, \& Mikulits, W. Initial steps of metastasis: cell invasion and endothelial transmigration. Mutat Res. 2011; 728: 23-24. doi:10.1016/j. mrrev.2011.05.002.

30. Naba A, Clauser KR, Hoersch S, Liu H, Carr SA, Hynes RO. The matrisome: in silico definition and in vivo characterization by proteomics of normal and tumor Extracellular Matrices. Mol Cell Proteomics. 2012; 11: M111.014647-M111.014647. doi: 10.1074/mcp. M111.014647.

31. Naba A, Clauser KR, Lamar JM, Carr SA, Hynes RO. Extracellular matrix signatures of human mammary carcinoma identify novel metastasis promoters. Elife. 2014; 2014: 1-23. doi: 10.7554/eLife.01308.

32. Tell RW, Horvath CM. Bioinformatic analysis reveals a pattern of STAT3-associated gene expression specific to basal-like breast cancers in human tumors. Proc Natl Acad Sci USA. 2014; 111: 1-6. doi: 10.1073/pnas.1404881111.

33. Das S, Harris LG, Metge BJ, Liu S, Riker AI, Samant RS, Shevde LA. The hedgehog pathway transcription factor GLI1 promotes malignant behavior of cancer cells by up-regulating osteopontin. J Biol Chem. 2009; 284: 22888 97. doi: 10.1074/jbc.M109.021949. 
34. Reddy TE, Pauli F, Sprouse RO, Neff NF, Newberry KM, Garabedian MJ, Myers RM. Genomic determination of the glucocorticoid response reveals unexpected mechanisms of gene regulation. Genome Res. 2009; 19: 2163-71. doi: 10.1101/gr.097022.109.

35. Love MI, Huber W, Anders S. Moderated estimation of fold change and dispersion for RNA-seq data with DESeq2. Genome Biol. 2014; 15: 550. doi: 10.1186/ s13059-014-0550-8.

36. Trapnell C, Pachter L, Salzberg SL. TopHat: Discovering splice junctions with RNA-Seq. Bioinformatics. 2009; 25: 1105-11. doi: 10.1093/bioinformatics/btp120.

37. Harrow J, Denoeud F, Frankish A, Reymond A, Chen C, Chrast J, Lagarde J, Gilbert JGR, Storey R, Swarbreck D, Rossier C, Ucla C, Hubbard T, et al. GENCODE: producing a reference annotation for ENCODE. 2006; 7: 1-9.

38. Trapnell C, Williams BA, Pertea G, Mortazavi A, Kwan G, van Baren MJ, Salzberg SL, Wold BJ, Pachter L. Transcript assembly and quantification by RNA-Seq reveals unannotated transcripts and isoform switching during cell differentiation. Nat Biotechnol. 2010; 28: 511-5. doi: 10.1038/nbt.1621.

39. Quinlan AR. BEDTools: The Swiss-Army tool for genome feature analysis. Current Protocols in Bioinformatics. 2014. 11.12.1-11.12.34 p. doi: 10.1002/0471250953.bi1112s47.

40. Zhang Y, Liu T, Meyer CA, Eeckhoute J, Johnson DS, Bernstein BE, Nusbaum C, Myers RM, Brown M, Li W, Liu
XS. Model-based analysis of ChIP-Seq (MACS). Genome Biol. 2008; 9: R137. doi: 10.1186/gb-2008-9-9-r137.

41. Bailey TL, Williams N, Misleh C, Li WW. MEME: Discovering and analyzing DNA and protein sequence motifs. Nucleic Acids Res. 2006; 34: 369-73. doi: 10.1093/ nar/gk1198.

42. R Core Team., 2015 R: A language and environment for statistical computing, $\mathrm{R}$ Foundation for Statistical Computing https://www.R-project.org.

43. Reddy TE, Gertz J, Crawford GE, Garabedian MJ, Myers RM. The hypersensitive glucocorticoid response specifically regulates period 1 and expression of circadian genes. Mol Cell Biol. 2012; 32: 3756-67. doi: 10.1128/MCB.00062-12.

44. Mootha VK, Lindgren CM, Eriksson K-F, Subramanian A, Sihag S, Lehar J, Puigserver P, Carlsson E, Ridderstråle M, Laurila E, Houstis N, Daly MJ, Patterson N, et al. PGC-1alpha-responsive genes involved in oxidative phosphorylation are coordinately downregulated in human diabetes. Nat Genet. 2003; 34: 267-73. doi: 10.1038/ ng1180.

45. Subramanian A, Tamayo P, Mootha VK, Mukherjee S, Ebert BL, Gillette MA, Paulovich A, Pomeroy SL, Golub TR, Lander ES, Mesirov JP. Gene set enrichment analysis: a knowledge-based approach for interpreting genome-wide expression profiles. Proc Natl Acad Sci. 2005; 102: 1554550. doi: 10.1073/pnas.0506580102. 